\title{
Les Soeurs Grises et les événements de 1869-1870
}

\section{Élisabeth De Moissac}

Volume 37, 1970

La vie religieuse au Manitoba

URI : https://id.erudit.org/iderudit/1007284ar

DOI : https://doi.org/10.7202/1007284ar

Aller au sommaire du numéro

Éditeur(s)

Les Éditions Historia Ecclesiæ Catholicæ Canadensis Inc.

ISSN

0318-6172 (imprimé)

1927-7067 (numérique)

Découvrir la revue

Citer cet article

De Moissac, É. (1970). Les Soeurs Grises et les événements de 1869-1870.

Sessions d'étude - Société canadienne d'histoire de l'Église catholique, 37 ,

215-228. https://doi.org/10.7202/1007284ar

Tous droits réservés @ Les Éditions Historia Ecclesiæ Catholicæ Canadensis Inc., 1972
Ce document est protégé par la loi sur le droit d'auteur. L'utilisation des services d'Érudit (y compris la reproduction) est assujettie à sa politique d'utilisation que vous pouvez consulter en ligne.

https://apropos.erudit.org/fr/usagers/politique-dutilisation/ 


\section{Les Sœurs Grises et les événements de $1869-1870$}

"Louis Riel est visiblement l'élu de Dieu pour sauver son pays. 》 Ainsi s'exprime la søur Curran ${ }^{1}$, dans une lettre adressée à la Maison Mère de Montréal, lettre où elle relate ce qui se passe sous ses yeux en cette fin d'année 1869.

Pouvons-nous blâmer cette religieuse si sincère de sa confiance en la mission de Louis Riel, le jeune chef métis? Je ne le crois pas, et je dis même que les sentiments qu'elle exprime ainsi ne sont que l'écho de ceux de ses compagnes, les vingt-cinq religieuses qui composaient alors la petite communauté de Saint-Boniface.

D'ailleurs, comment en aurait-il été autrement puisque, depuis 1844, les Sœurs Grises, premières missionnaires, éducatrices et hospitalières de l'Ouest canadien, partageaient les joies et les peines de leurs chers Métis, leur genre de vie même, en un certain sens, puisqu'elles se contentaient de leur menu de viande sèche et se chaussaient comme eux de mocassins.

Nos chers Métis, ces mots reviennent fréquemment sous la plume de Mère Valade ${ }^{2}$, première supérieure de la maison. Elle les connaît bien et elle n'ignore pas leur incurie, mais elle s'inquiète sans cesse de la grande pauvreté qui règne autour d'elle. Elle écrit:

La misère est très grande dans ce pays; tout le monde a semé autant de blé qu'il a pu, et même plus, car beaucoup ne se sont rien réservé pour leur nourriture, aimant mieux vivre de pêche et de chasse que de confier à la terre le peu de grain qu'ils possèdent ${ }^{3}$.

Et un mois plus tard elle ajoute:

Un grand nombre de nos gens jeûnent fréquemment. Heureusement que les chasseurs de bisons vont arriver. Nous aussi nous avons hâte,

1 La sœur Mary A. Curran (1831-1906). Professe de notre communautésœur de Bytown. Cédée à la petite communauté de la Rivière Rouge en 1853. Sa parfaite connaissance des langues française et anglaise lui permit de servir de secrétaire à $M^{\mathrm{gr}}$ Taché qui ne pouvait immobiliser un de ses prêtres à ce poste. Les sœurs de Saint-Boniface doivent à la sœur Curran une part importante de leurs archives. Elle fut rappelée à Montréal en 1887 .

2 La sœur M.-Louise Valade, fondatrice de la mission de la Rivière Rouge, née le 27 décembre 1808, à Sainte-Anne-des-Plaines, décédée à Saint-Boniface le 13 mai 1861.

3 Lettre de Mère Valade à Mère Deschamps, 30 juin 1853. Archives, Maison Provinciale, Saint-Boniface, Man. [désormais Maison P.S.B.]. 
car si nous ne sommes pas rendues à jeûner, nous n'en avons pas de reste ${ }^{4}$.

Ces chasses. quelle place elles occupaient dans ce pays où le pemmican était le régime quotidien! Une chasse malheureuse conduisait à la disette.

Ailleurs, les sœurs se réjouissent de la célèbre victoire que leurs chers Métis viennent de remporter sur les Sioux, les 13 et 14 juillet 1851, à la bataille du Grand Coteau du Missouri.

Après une courte description de la rencontre, la sœur Lagrave ${ }^{5}$. compagne de Mère Valade et alors à la Prairie du Cheval Blanc, SaintFrançois-Xavier, conclut par ces mots:

Voyant qu'ils n'avaient aucun succès, les Sioux prirent le parti de se retirer, laissant nos Métis bien joyeux de leur retraite. Il est certain que nos gens n'auraient jamais été capables de se tirer d'affaire sans une protection spéciale ${ }^{6}$.

Toutefois, si un tel succès exaltait la fierté de ces hardis descendants des Voyageurs, une autre victoire, remportée deux ans auparavant sur le monopole de la traite, les remplissait d'aise.

Depuis longtemps, les habitants de la colonie s'insurgeaient contre les règlements rigoureux imposés par la Compagnie de la Baie d'Hudson, en vertu de sa charte, et encore plus des vexations continuelles de certains fonctionnaires.

De son côté, le gouvernement de l'Assiniboia, alarmé par une pétition envoyée à Londres, en 1848, pour réclamer la liberté du commerce, avait dépêché une petite troupe à la Rivière Rouge.

Voici ce qu'écrit à ce sujet Mère Valade:

Il nous est arrivé, cet automne, un nouveau gouverneur du nom de Caldwell, avec un petit régiment [...]. In n'a encore rien changé de la Compagnie; il paraît même qu'il est pour maintenir le même système. Nous pensons que la Compagnie fait venir des soldats pour jeter de la poudre aux yeux des Métis; je ne sais s'ils se laisseront aveugler 7.

Ils ne le furent point puisque, quelques mois plus tard, le 17 mai 1849, jour de l'Ascension, ces braves, ayant Louis Riel, père, à leur tête, arrachaient des mains de l'Honorable Compagnie, non seulement deux des leurs, inculpés dans une affaire de traite, mais aussi un fort lambeau de son autorité souveraine.

\section{Ibid.}

5 La sœur Eulalie Lagrave, née le 2 mai 1805, à Saint-Charles, sur la rivière Chambly. Assistante de Mère Valade dès 1844, elle visite les pauvres et les malades à domicile. En 1850, elle fonde la deuxième mission des Sœurs Grises à la R.R.: Saint-François-Xavier. Elle est décédée à Saint-Boniface, le 4 août 1859.

6 Archives, Maison P. S.B., juillet 1851.

7 Lettre de Mère Valade à Mère McMullen, 27 novembre 1848. 
Un certain Reverend, de la secte anglicane, fut alors fort choqué de ce coup d'audace, préparé avec soin par les habitants du pays. Dans son Journal, il accuse nos sœurs ainsi:

It is also a well known fact that for some time the nuns [les Sœurs de Charité] have gone from home to home lecturing the women to use their influence with their husbands in endeavouring to defend what they term the rights of the H.C. 8.

Amusante accusation, car il est bien permis de douter que nos sœurs aient eu le temps de battre la campagne, même en faveur de leurs amis. Elles n'étaient pas nombreuses à cette époque et fort occupées à l'aménagement de leur nouveau logis. Puis aller de maison en maison n'était pas si facile, les habitations s'échelonnaient tout le long de la rivière, reliées entre elles par des sentiers. Toutefois, les sœurs ne pouvaient pas rester indifférentes aux justes revendications des habitants. Elles écrivent:

Des suppliques incessantes s'étaient élevées tout le jour vers le ciel pour obtenir sans effusion de sang la solution de cette épineuse affaire. Aussi, le soir, tous les cœurs entonnèrent l'hymne de la reconnaissance $\boldsymbol{\theta}$.

Soulignons ici que le nom du brave Métis qui s'était distingué ce jour-là revient souvent dans notre histoire familiale. Chose bien naturelle, puisque $M$. Riel fut toujours pour les sœurs un ami sincère et dévoué.

Première coïncidence, ce fut peu après l'arrivée des sœurs que naquit, le 22 octobre 1844, Louis, le fils aîné de Louis Riel, dit l'Irlande, et de Julie Lagimodière. Et lorsqu'il fut question de donner à l'enfant une éducation plus soignée, ce fut à Mère Valade, en route pour la Maison Mère de Montréal, que $\mathrm{M}^{\mathrm{gr}}$ Provencher confia Louis et deux autres jeunes Métis. Disons, en passant, que ce fut avec un soupir d'aise que la bonne supérieure remit la trio en mains sûres. Une fois entrés en pays civilisé, ces jeunes sauvageons devenaient incontrôlables.

Ce fut également à M. Riel, le Meunier de la Seine, que les Sœurs Grises, tisserandes par nécessité, s'adressèrent pour rendre moins pénible le cardage de la laine qu'elles utilisaient pour se fabriquer des étoffes. Voici ce que l'on rapporte en 1855 :

Dans les derniers jours de juillet, nous recevions une machine à carder. Deux hommes à l'aide de manivelles sont requis pour la manœuvrer. Alors, Mère Valade fit des démarches près de $M$. Riel pour qu'il ajuste notre machine à son pouvoir d'eau. Ce bon monsieur y consentit volontiers et n'épargna ni son temps ni sa peine pour nous rendre service. Cependant, comme il lui était impossible d'être cardeur

8 * Smithurst ", Journal from the Church Missionary Society, John SMITHURst, May 16, 1849. Also T. B. C. Boon, The Anglican Church from the Bay to the Rockies, Ryerson Press, p. 47. (C'était pour lui un fait bien connu que les Seurs de Charité étaient allées de porte en porte, exhortant les femmes à encourager leurs maris à tenir tête à la Compagnie.)

9 Archives, Maison P. S.B., 1855. 
et meunier en même temps, sœur Cusson se rendait en voiture au moulin

de M. Riel, accompagnée d'une fille, pour y carder notre laine. Elles nous revenaient tous les soirs pour repartir tous les matins; ce travail durant deux ou trois semaines 10 .

Si M. Riel accueillait ainsi charitablement les søurs cardeuses à son moulin, il estimait aussi grandement la sœur Sainte-Thérèse ${ }^{11}$, car cette bonne religieuse, à la suite de la sœur Lagrave, était devenue la providence du pays. Tous, jeunes et vieux, l'appelaient " notre sœur docteur ".

Aussi, quelle ne fut pas la consternation de tous ces pauvres gens, lorsque la sœur Sainte-Thérèse, prêtée par notre communauté-sœur de Bytown, pour aider les fondatrices, fut rappelée par ses supérieures, en 1857 !

Qui est-ce qui nous soignera si notre docteur s'en va? fut le cri général. Des paroles aux actes, il n'y a qu'un pas: sous la conduite de $M$. Riel, les Métis résolurent de conserver à la colonie une femme si précieuse. Au premier campement, la sœur Saint-Thérèse fut cernée, invitée à monter dans une charrette conduite par Céleste Lagimodière ${ }^{12}$, nièce de M. Riel, et forcée de reprendre le chemin de Saint-Boniface. Quelle entrée triomphale firent ces braves ramenant « la sœur docteur", nullement fâchée de rester parmi eux !

1861, année terrible pour les pauvres habitants de la Rivière Rouge, le pays est inondé. Les religieuses, enfermées au second étage de leur maison, ont en même temps la douleur de voir mourir dans leurs bras leur chère Mère Valade.

A leur chagrin bien naturel, s'ajoutait l'impossibilité de suivre la dépouille mortelle de leur fondatrice jusqu'à sa dernière demeure, le sol étant encore couvert d'un pied d'eau. Quatre hommes robustes, dont M. Riel, transportèrent le cercueil jusqu'aux ruines de la cathédrale où $\mathbf{M}^{\mathrm{gr}}$ Taché avait fait creuser la fosse sous les débris du clocher.

L'ami si fidèle qu'avait toujours été M. Riel méritait bien d'avoir, à son chevet d'agonie, ses bonnes Sœurs Grises. Voici comment sont relatés dans nos chroniques les derniers moments du Meunier de la Seine:

Depuis le premier de l'an, nos søurs infirmières entourèrent de tous les soins possibles, M. Louis Riel, et demeurèrent près de lui le jour et

10 Archives, Maison P. S.B., 1855.

11 La sœur Sainte-Thérèse, Margaret Teresa McDonald, née en 1835 à Saint-André d'Ontario, entrée au noviciat des Sœurs Grises de Bytown, le 31 janvier 185l. Venue à la Rivière Rouge en 1855 , elle y demeura jusqu'à sa mort, le 4 novembre 1917. Elle fut la première supérieure de l'Hôpital de Saint-Boniface, fondé en 1871.

12 Céleste Lagimodière, fille de Benjamin et d'Angélique Carrière. Elle épousa Octave Allard. 
la nuit. Mais tout fut inutile, le 21 janvier, elles étaient témoins de ce que sont les derniers moments d'un fervent chrétien 13.

\section{Bel éloge et bien mérité.}

La disparition du grand meneur de jeu de la lutte pour le commerce libre marque une étape dans l'histoire de la colonie. La mission du père est terminée, mais bientôt commencera celle de son fils, Louis. Tâche plus grande encore parce que plus lourde de conséquences.

Ici, nous entrons enfin dans le cœur de notre sujet: les événements qui ont précédé la naissance du Manitoba comme province. Toutefois, il nous a semblé nécessaire, avant de l'aborder, d'insister sur les relations très étroites qui existaient entre les Sœurs Grises et les habitants de la Rivière Rouge et, en particulier, avec la famille Riel.

Déjà, depuis 1857, il était question de relier la colonie au Canada par une route, autre que celle qui passait par les États-Unis. Les missions de Dawson-Hind, pour le Canada, et de Palliser pour l'Angleterre vinrent enquêter à cet effet. La sœur Lagrave informe ainsi sa supérieure de Montréal:

Savez-vous que dans le moment, tout le Canada est en rumeur au sujet de notre pauvre pays. On parle fortement d'annexion; un monsieur a été envoyé pour cette affaire et, ce dit monsieur, assure qu'aussitôt l'affaire conclue, on se mettrait à l'œuvre pour construire un chemin de fer qui s'embrancherait à ceux du Canada. Tout cela paraît bien extraordinaire ${ }^{14}$.

Le projet n'aboutira pas immédiatement. Toutefois, deux ans plus tard, le premier bateau à vapeur, le Northup, fait son apparition dans les eaux de la Rouge.

Ce fut tout un événement: les cloches de la cathédrale firent entendre leurs joyeuses volées pour saluer l'arrivée de ce vapeur, qui n'était pas certes un chef-d'œuvre du genre. Tout le monde accourait sur la rive pour considérer ce canot monstre. Nos Indiens ne lui souhaitèrent pas la bienvenue, car disaient-ils, bien sûr cette épouvantable machine chassera les poissons de nos rivières. Nos canots et nos barges nous suffisent 15 .

Toutefois, en 1868, les projets d'une route se réalisèrent avec le chemin Dawson.

Un monsieur Snow est arrivé dans le pays pour surveiller les travaux du chemin qui doit partir d'ici pour se rendre du côté du lac Supérieur; ceux qui pourront ou voudront s'engager pour cette entreprise y trouveront des avantages, surtout pendant cette année de misère. On offre trois louis en argent, et la nourriture à part. Il me semble avoir entendu dire qu'on faisait venir des provisions à cet effet par le lac Supérieur. Il serait impossible de se les procurer ici 16.

13 Archives de la Maison P. S.B., 22 janvier 1864.

14 Lettre de la sœur Lagrave à Mère Deschamps, 28 mai 1857.

15 Archives, Maison P. S.B., 10 juin 1859.

16 Archives, Maison P. S.B., $1^{\text {er }}$ décembre 1868. 
La chronique omet de décrire les tribulations des pauvres habitants de la Pointe-des-Chênes, bernés par Snow et ses acolytes. Elle ne mentionne pas non plus les vexations des arpenteurs, empiétant sur les terres des habitants. Il est vrai que nos sœurs n'ouvriront leur école à Sainte-Anne-des-Chênes qu'en 1883 .

Et pour cause, puisque les événements se précipitent et qu'une guerre civile semble imminente. C'est alors que la sœur Curran, dans un long rapport à ses supérieures de Montréal, notera presque jour par jour, et dans le style du temps, les incidents de ces heures tragiques.

Dès le mois de juin dernier, on annonçait, presqu'à chaque arrivée, que le Gouverneur McDougall devait prendre possession du territoire acquis par le Canada; cette acquisition avait été faite sans que les habitants du pays fussent consultés. Ils en furent froissés, mais ce ne fut que lorsque les pauvres gens apprirent que, très probablement, leur religion serait persécutée, qu'ils résolurent de prendre la défensive et à repousser l'agresseur. La première intervention du ciel est bien manifeste, en ce que, si Monsieur McDougall était parti au mois de juin, il y avait trop d'hommes absents, soit dans les barges ou à la chasse, qu'on aurait osé compter sur le succès; mais au mois d'octobre, plusieurs centaines étaient revenus ${ }^{17}$.

Elle fait ensuite allusion aux efforts du parti Canadien, la plupart gens venus de l'Ontario pour se saisir des terres, pour gagner les Métis au projet d'annexion de la colonie avec le Canada.

Les Canadiens et autres intéressés firent de leur mieux pour paralyser les efforts des Métis catholiques. Ils firent circuler des papiers, cherchant des signatures pour faire opposition. Dans ces circonstances, on eut à admirer la foi et la fermeté de plusieurs de nos pauvres gens. Je vous en citerai un entre autres qui répondit à l'offre qu'on lui faisait de lui donner 10 louis s'il voulait signer: a Pas pour 10 louis, ni pour 1000 louis que je voudrais vendre mon âme: je suis le plus pauvre de la paroisse, tout le monde le sait, mais $j$ 'aime mieux souffir que de faire ce que vous me demandez. Si vous voulez aller en enfer, vous pouvez y aller sans moi !.

Des pauvres mères restaient à la maison sans bois et sans provisions pour laisser partir leur mari et leurs fils, afin de défendre la bonne cause; puis s'adressant à leurs petits enfants: . Vous n'êtes pas assez grands pour aller combattre, mais du moins prions et souffrons notre misère pour le bon Dieu ${ }^{18}$,

\section{Combien naïfs mais sincères étaient ces gens simples et bons.}

Sous le coup de telles menaces s'ouvre ainsi une période d'alertes continuelles qui créent un climat d'angoisse indescriptible. non seulement parmi les habitants, mais aussi dans le cœur des religieuses de SaintBoniface qui n'ont pour toute arme que la prière.

Le bruit court que le gouverneur arrive avec soixante hommes armés de carabines à seize coups; armes bien supérieures à tout ce que possédaient nos pauvres Métis. N'importe, ils partent après s'être rendus

$1 \bar{i}$ Rapport de la sœur Curran à la Maison Mère de Montréal sur les événements de 1869-1870, 21 septembre 1869.

18 Ibid., 21 septembre 1869. 
forts par la réception des sacrements qu'ils recevaient comme préparation à la mort qui paraissait inévitable pour plusieurs. Deux jours plus tard, on vient crier d'éteindre toutes les lumières, qu'on tirait sur l'évêché et chez-nous. Le courrier avait d'abord sonné à la communauté, mais ne recevant pas de réponse assez prompte, il avait couru au réfectoire. La nouvelle des soixante hommes était fausse, mais pendant trois jours et trois nuits, le Saint Sacrement était exposé dans notre chapelle, et chacune y allait de ses dévotions, jusqu'à cinq ou six rosaires par jour. Monsieur Dugas [Georges] était aussi à la chapelle et conjurait avec larmes le Seigneur d'avoir pitié de nous ${ }^{19}$.

Ici, qu'il nous soit permis d'insérer un extrait d'une lettre publiée dans un journal de Montréal et envoyée de la Rivière Rouge le 17 décembre 1869:

...Si maintenant vous voulez savoir la part que notre clergé a prise dans ce mouvement, le voici...

Quant à la part prise dans l'affaire par les Sœurs de la Charité, eille se réduit à une exposition du Saint Sacrement, dans la petite chapelle où, pendant soixante-dix heures consécutives, ces saintes religieuses sont venues prier et pleurer nos malheurs.

$$
\text { signé: Un habitant du Nord Ouest } 20 .
$$

Qui est l'auteur de cet article ? On ne le dit pas. Quelles étaient ses intentions? Peut-être tout simplement d'exonérer les religieuses de toute activité portant ombrage à l'autorité établie... c'est-à-dire d'encourager les Métis à la révolte, révolte qui n'en était pas une, mais qui passait comme telle à certains yeux. Il se trouvait peut-être encore des "Smithurst " pour calomnier ceux et celles qui prenaient parti pour la cause des habitants du pays.

Nous ne croyons pas qu'elles aient réalisé tout cela, mais nous lisons dans leur correspondance de l'époque le passage suivant:

Pour la première fois de notre vie, nous remercions le bon Dieu que Monseigneur ne soit pas ici; dans quelle position critique il se trouverait. Pauvre évêque, qu'il est loin de penser ce qui se fait ici ${ }^{21}$.

\section{Monseigneur Taché, on le sait, était alors à Rome.}

Ce ne fut que plus tard, reprend la sour Curran, que l'on apprit ce qui s'était passé, ce 28 octobre. Le parti opposé, les Canadiens, avaient voulu aller surprendre et massacrer les Métis retranchés dans les bois de Saint-Norbert. Des espions étaient partis en éclaireurs... Mais Dieu veillait sur les siens, qui n'étaient qu'un très petit nombre ce soir là. A peine les espions étaient-ils à quelque distance de SaintNorbert, que cinq d'entre eux se réfugièrent fort effrayés dans la maison d'un métis de l'endroit... disant que le plus prudent était de s'éloigner, qu'ils y avaient vu des milliers de Métis de rassemblés. Le brave homme surpris, leur dit qu'il n'y avait personne sur le chemin où ils devaient passer; mais eux reprirent: - Vous vous trompez, le bois en était plein, il y a autant d'hommes que d'arbres!. Alors, il les reconduisit jusqu'à ce qu'ils fussent hors de danger. Personne ne doute ici que le bon Dieu ait voulu accorder une protection toute visible à son peuple 22 .

19 lbid., 26 octobre 1869.

20 L'Ordre, Montréal, numéro du 13 janvier 1870.

21 Ibid.

22 lbid. 
Les chers Métis, si près de la nature, étaient souvent hantés par des rêves, des apparitions et autres manifestations miraculeuses. Il ne faut donc pas reprocher à la sœur Curran, leur fidèle interprète, de croire un peu au merveilleux et à un petit miracle en faveur de la cause.

Quant aux Métis, ils étaient réellement embusqués à Saint-Norbert et bien décidés d'empêcher le gouverneur McDougal d'entrer dans la colonie. Dans une clairière, près de la rivière Sale, ils avaient construit une "barrière " solide faite de troncs d'arbres jetés à travers de la piste et de charrettes liées ensemble, le tout bloquant parfaitement la voie que devaient suivre le gouverneur et sa suite pour atteindre le Fort Garry. Des hommes armés y veillaient sans relâche. Comme on était à la fin d'octobre et qu'il faisait très froid, ces braves, qui ne pouvaient pas rester jour et nuit dans les bois, venaient à tour de rôle se chauffer au couvent, où on leur servait du thé bien chaud et de la galette. Ceux qui n'étaient pas de garde « bivouaquaient dans l'église paroissiale ${ }^{23}$ ».

Est-il nécessaire de rappeler que deux Sœurs Grises y tenaient l'école depuis 1858 et que leurs chers Métis y étaient chez eux? Il est aussi intéressant de noter que la compagne de la sœur Dussault, supérieure, se trouvait alors une très jeune religieuse, qui n'était autre que Sara Riel, fille du Meunier de la Seine et sœur cadette de Louis. Sara était entrée au noviciat de Saint-Boniface, le 2 septembre 1865, peu de temps après la mort de son père.

Nous ne dormions pas, racontait plus tard la sœur Dussault, nous ne pouvions pas dormir, car nous ne savions pas à quelle heure arriverait le gouverneur. La bonne ancienne confia un jour à une intime (notre sœur Anna Bonin) que le tremblement fébrile qui secoua son pauvre corps jusqu'à la fin de sa vie n'eut d'autre cause que les émotions de ces jours d'angoisse en pleine zone de guerre.

Enfin, le $1^{\text {er }}$ novembre, après bien des jours d'alertes, les Métis virent apparaître des cavaliers. Non pas McDougall lui-même qui, prudemment et avec raison, était resté à Pembina, mais ses éclaireurs: M. J. N. Provencher et, quelques heures plus tard, le capitaine Cameron.

Si M. Provencher fut reçu au presbytère par M. Ritchot, curé de la paroisse et l'ami de Riel et des Métis, Cameron, lui, dut tourner bride et reprendre le chemin de Pembina. Dans son rapport au gouverneur, il dut l'assurer que les Métis étaient des gens résolus et que l'on ne saurait les mésestimer. Et, cependant, pas un coup de feu n'avait été tiré devant la Barrière.

23 John H. O’Donnelu, Manitoba as I Saw It, From 1869 to date, Musson, 1909, p. 23. 
Peu après, d'autres voyageurs apparurent à leur tour devant l'obstacle: M. et Mme Mair, de retour de leur voyage de noces aux États-Unis, et les O'Donnell. Ce dernier était un jeune médecin qui venait s'établir dans le pays avec son épouse. Arrêtés et questionnés par les patriotes, ils furent conduits sous escorte jusqu'au village de Saint-Norbert.

Pendant que M. Ritchot donnait l'hospitalité aux messieurs, les dames étaient reçues avec bonté par les sœurs qui leur offrirent tout le confort que leur permettait leur grande pauvreté ${ }^{24}$. Mme Mair, nièce du docteur Schultz, était bien connue dans le pays et les 0'Donnell, inoffensifs. Après quelques jours, ces voyageurs purent poursuivre leur route jusqu'au Fort Garry.

Pour perpétuer le souvenir de la Barrière, l'Union Métisse érigea d'abord une simple croix de bois, le 24, octobre 1906. Cette croix fut remplacée, en 1922, par le monument actuel, que l'on peut voir en bordure de la route 75 , non loin du pont de la rivière Sale ${ }^{25}$.

Pendant que ces derniers événements se déroulaient à Saint-Norbert, Louis Riel, à la tête d'une cinquantaine d'hommes résolus, s'emparait, le 2 novembre 1869, du Fort Garry dont les gardiens n'offrirent aucune résistance. Les Métis y trouvèrent tout un assortiment d'armes, prise bien utile en la circonstance.

La sour Curran, après avoir relaté le fait, continue ainsi:

Depuis ce temps, une forte garde y demeure jour et nuit, mais il reste encore beaucoup à faire et à déjouer. Des découvertes y furent faites, puisqu'en temps de guerre les lettres peuvent être ouvertes. De sévères mesures furent prises: personne ne pouvant passer sans prêter serment de fidélité; on fouillait les caravanes et on confisqua le magnifique mobilier qui arrivait avec le gouverneur ainsi que toutes les armes 26 .

C'est admirable, ajoute-t-elle, de voir les dispositions de nos bons Métis. Leur plus grande crainte était d'être obligés de verser le sang de leurs semblables, aussi, jusqu'à présent, pas une goutte de sang n'a été répandue. Ce qui est certain, c'est que Dieu conduit et protège nos paurres gens.

Elle fait ensuite un bel éloge du jeune chef métis:

Louis Riel est visiblement l'élu de Dieu pour sauver son pays; il se regarde comme le faible instrument de la providence et fait l'admiration de tous. Il se met toujours au premier poste lorsqu'il y a danger et se tient prêt à la mort, à laquelle il s'attend à tout moment ${ }^{27}$. En effet, parmi les complots qui se découvrent, continue la narratrice, deux individus ont dit qu'on leur offrait une grosse somme pour tuer

24 ID., ibid., p. 23.

25 Archives de l'Union Métisse, société fondée en 1887, ici au Manitoba, mais fondée en 1884 à Batoche.

26 Rapport de la sœur Curran, 2 novembre 1869.

27 Ibid. 
Monsieur Bruce, qui est président du Gouvernement Provisoire et Riel qui est son secrétaire 28 .

Le danger qui menaçait la vie de Louis Riel et de ses amis était réel et alarmait les leurs. De Saint-Norbert la sœur Sara Riel écrivait à sa mère:

Chère maman, je vous prie de recommander à ce cher frère d'être prudent, de ne pas marcher seul, la nuit, il peut y avoir du danger à le faire. Mon cœur est tantôt en proie à la crainte et à l'inquiétude et tantôt bercé par l'espérance; quelque chose me dit d'espérer et il me semble que je suis sûre qu'il réussira 29 .

Pauvre petite Sara, elle eut toujours foi en la mission de son frère.

La veille de l'Immaculée-Conception, les Métis cernèrent la maison du docteur Schultz, chef du parti Canadien, et firent prisonniers les occupants. La sœur Curran relate le fait, véridique en lui-même, mais qui, lui ayant été raconté par les voies ordinaires de la " petite presse locale ", est en quelque sorte dramatisé et agrémenté de toute la saveur de l'imagination d'un brave, un peu vantard et sûrement très fier de la victoire des siens:

Je vous donne quelques détails sur la prise des prisonniers: un individu de la pire espèce [le $\mathrm{D}^{\boldsymbol{r}}$ Schultz], qui est dans le pays depuis plusieurs années, a fait plusieurs voyages au Canada; il avait réuni chez lui trente personnes, toutes armées jusqu'aux dents. On le somma de se livrer, autrement on ferait sauter la maison. Vingt-quatre canons [sic] étaient braqués en conséquence; sa demeure était entourée d'une forte garde, mais il ne se rendit pas.

Le 7 décembre, on y retourna; ces malheureux dirent avoir pris une potion pour se rendre plus braves, qui malheureusement eut un effet tout contraire; à l'approche des Métis, les bras leur tombèrent, ils livrèrent toutes leurs armes, et se laissèrent conduire en prison.

On fit la visite de cette maison, qu'on appelle à juste titre * maudite ", on y découvrit des armes à feu chargées, jusque dans les tuyaux des poêles. Avant de partir pour la prison, ils avaient éteint tous les feux, puis placé des sacs de poudre sous la cendre, une étincelle aurait suffi pour faire sauter une partie de la ville. Nous voyons cette maison très distinctement d'ici, et elle est voisine de notre maison d'école de la ville ${ }^{30}$.

En effet, tout se passait autour du Fort Garry, de l'autre côté de la rivière, presque en face de leur couvent et les sœurs pouvaient facilement percevoir l'agitation continuelle des soldats métis autour du Fort et des environs.

N'est-ce pas que la protection de Dieu est visible, poursuit la chroniqueuse. Des conversions nombreuses se sont opérées à l'occasion de ces troubles. Des hommes qui ne s'étaient pas confessés depuis plusieurs années se sont rendus avant d'aller se battre. La foi de notre peuple se manifeste de plus en plus. Leur clémence pour les vaincus leur fait honneur. Ils traitent les prisonniers avec égards, et les ordres

28 Ibid.

29 Lettre de la søur Sara Riel à sa mère. Saint-Norbert, 18 octobre 1869.

30 Rapport de la sceur Curran, décembre 1869. L'école Sainte-Marie de Winnipeg fut fondée par nos søurs le $1^{\text {er }}$ mai 1869. Plus tard, elle fut cédée aux Sœurs des Saints Noms de Jésus et Marie, en 1874. 
des chefs à ce sujet sont formels. On ne sait quel sera le sort de ces infortunés, leur procès doit passer ces jours-ci 31 .

Le 19 janvier, la sœur Curran reprend son récit, car il semble que le jour de Noël et le $1^{\text {er }}$ janvier 1870 fussent relativement calmes.

Donald Smith est arrivé d'Ottawa, armé de documents qui nécessitent une convention générale. Quarante délégués des deux langues se réunissent et nomment Louis Riel Président.

Ce soir là, vers dix heures, on entendit des coups de canon, puis des cris de joie. On fit un magnifique feu d'artifice ${ }^{32}$.

Cependant, les alertes se succèdent au cours des semaines qui suivent, plusieurs prisonniers s'étaient évadés et cherchaient à opérer une jonction avec les Ontariens du Portage.

On vient nous avertir que les gens du Portage arrivent pour mettre en liberté les autres prisonniers, le 14 on les croit repartis, mais le 15 , dans la nuit, ils passent nrès du Fort et vonnt entourer la maison de Riel. Il n'y était pas heureusement ${ }^{33}$.

Le jeune chef métis logeait ordinairement chez un ami, un nommé Henri Coutu.

Nouvelle alarme: les Sioux arrivent, dit-on, pour se rendre à l'invitation que leur ont fait les Anglais. Comme on dit qu'il y aura de la guerre aujourd'hui. Toutes les femmes et les enfants sont partis pour se mettre en sûreté; quelques parents viennent chercher leurs enfants au pensionnat.

Enfin, on nous annonce que les Sioux n'avanceront pas, voyant qu'ils ont été trompés.

A peine respirions-nous un peu, qu'on vient nous avertir que les Anglais viendraient ce soir même, s'emparer de la cathédrale et de l'évêché, pour se faire un lieu fortifié. Aussitôt, il faut ôter de l'église ce qui pourrait être exposé à la profanation; puis les soldats ôtèrent les poêles afin que les ennemis eussent froid pour charger leurs fusils longtemps.

Nous avions fait chacune notre petit paquet, car on croyait possible qu'on chercherait à mettre le feu chez-nous. Celles qui n'avaient pas la force de veiller devaient se coucher tout habillées.

Vers neuf heures, le bon Dieu a eu pitié de nous et encore une fois il a fait triompher sa puissance. On vint nous dire que les ennemis se dispersaient. Quarante soldats montaient la garde à l'évêché et des cavaliers faisaient le tour en tous sens pour découvrir si quelqu'un apnrochait. Le Saint Sacrement a été exposé dans notre chapelle, le jour et la nuit.

Le lendemain, vers neuf heures, de nouveaux cris se font entendre, puis on voit partir des cavaliers en toute hâte; il faut voir les Métis à cheval pour s'en faire une idée; ils avaient aperçu au loin, les gens du Portage qui cherchaient à s'en retourner et ils les poursuivaient. Arrivés près d'eux, un cavalier s'avance et leur dit d'arrêter: tout tremblants, ils obéissent, déposent les armes et sont amenés au Fort et faits prisonniers. Je ne sais quel sera leur sort ${ }^{34}$.

31 Rapport de la sœur Curran.

22 Ibid., 19 janvier 1870 .

33 Ibid., 13 février 1870.

34 Ibid., 17 février 1870. 
Que doit-on penser de toutes ces alertes et frayeurs par lesquelles sont passées nos pauvres devancières en ces années 1869-1870. Le danger était-il réel ou grossi par l'imagination surexcitée de leurs amis métis ? Sans doute, mais ce qui est certain, c'est que les Canadiens, partisans de l'annexion pure et simple, étaient bien décidés à gagner leur cause. Ils méprisaient les Métis qu'ils jugeaient très inférieurs.

Le docteur Schultz, en tant que médecin, s'était montré charitable au début de son séjour dans la colonie. Nos sœurs avaient même eu à se louer de lui lors de leurs visites aux pauvres. Mais, par contre, à l'époque que nous décrivons, il était devenu l'ennemie Numéro Un. Quant aux gens du Portage, la plupart aventuriers en quête des meilleures terres du pays, ils étaient prêts à tout pour arriver à leurs fins.

Toutefois ils eurent à compter avec les Métis, adroits tireurs, qui manquaient rarement leur bison. Au cours de leurs expéditions de chasse ils étaient soumis à une certaine discipline et en 1869-1870, ils avaient à leur tête un jeune chef, intelligent et résolu, sûr de la légitimité de la cause qu'il défendait.

Quant à l'intervention des Sioux les historiens ne sont pas précis sur leurs intentions et leurs mouvements. Il devait quand même y avoir un certain fondement à cette rumeur, car elle causa une certaine panique au sein des familles. Les atrocités de leurs massacres en terre américaine étaient dans toutes les mémoires.

Puis nos chères devancières avaient charge d'âmes; leur maison était pleine d'orphelines, 45 en 1869, de pensionnaires et de femmes âgées. Elles eurent sûrement très peur, plus de peur que de mal, mais peut-on les blâmer de trembler un peu chaque fois que leurs amis métis venaient leur faire part d'un nouveau danger?

Avec les beaux jours, nos sœurs eurent un peu de répit. Le 13 mars 1870, Mère Clapin, troisième supérieure de la Rivière Rouge, écrivait:

Les provisions, les grains sont à bon marché, malheureusement nous n'avons pas d'argent pour en acheter et pas moyen, à présent, d'en obtenir de la Compagnie. Je ne sais ce que nous ferons l'été prochain, car il est probable que les Métis n'iront pas à la chasse. Selon les apparences, ils resteront pour défendre le pays 35 .

A son tour, la sœur Curran écrit le 19 avril suivant:

Pour le présent, nous jouissons d'une paix et d'une tranquilité qui soulagent tout le monde. Après l'hiver que nous avons passé, il semble que nous revenons à la vie. Pourtant, on nous dit que nous avons besoin de continuer à beaucoup prier ${ }^{36}$.

La signature à Ottawa de l'Acte du Manitoba, qui faisait de la colonie de la Rivière Rouge une province du Canada, ne sembla pas

35 La sœur Clapin à Mère McMullen, 13 mars 1870.

36 La sœur Curran à la Maison Mère, 19 avril 1870. 
avoir suscité beaucoup d'enthousiasme ici, autrement nos chroniques y auraient fait allusion. Par contre, le 26 juillet, la sœur Curran annonce l'arrivée prochaine du nouveau gouverneur, Adams G. Archibald.

Après un hiver de transes, on entend parler à présent de préparatifs de réception pour Son Excellence, qui doit arriver, dit-on, vers la mi-août. Il paraît que nos bons Métis vont faire de leur mieux et que déjà, plusieurs sont partis à sa rencontre jusqu'à Alexandria ${ }^{37}$.

Toutefois, alarmées par l'agitation des esprits qui avait régné au cours de l'hiver, les autorités d'Ottawa avaient dépêché un corps expéditionnaire, afin de mettre fin à la situation.

Par une pluie battante, le 24 août 1870 , la troupe régulière vint prendre possession du Fort Garry, mettant ainsi fin au Gouvernement Provisoire.

Par suite des misères et des privations de leur long voyage, plusieurs soldats tombèrent malades en arrivant. Alors, se souvenant sans doute de la charité de notre Bienheureuse Fondatrice, Mère d'Youville, pour les soldats prisonniers de son temps, Mère Clapin offrit les services des sœurs au colonel Wolseley pour les malades de son régiment. Il accepta avec empressement et reconnaissance.

Nous avons commencé à les visiter le 3 septembre, dit la chronique, depuis nous y allons tous les jours. Le colonel Wolseley, le capitaine W. Butler et le premier médecin, tous sont venus nous rencontrer, pour nous exprimer leur gratitude et leur surprise pour un tel acte. Les sœurs durent même se rendre jusqu'au Fort de Pierre, Lower Fort Garry, pour cette mission charitable ${ }^{38}$.

Néanmoins, l'arrivée des soldats qui aurait dû, ce semble, ouvrir une ère de paix pour toute la province fut, du moins pour les Métis, une période de persécution intense.

La haine de ces Ontariens poursuivait tous ceux qu'ils croyaient être les meurtriers de Scott et, parmi ces derniers, Louis Riel. Aussi, sachant que Louis venait, au péril de sa vie, voir sa mère à Saint-Vital, la pauvre Julie fut victime de visites domiciliaires répétées.

Cette poursuite du jeune chef métis s'étendit également à la région de Saint-Norbert où, on le savait, M. Ritchot lui donnait souvent l'hospitalité. Voici ce que l'on peut lire dans un journal de l'époque: "Ces énergumènes, toujours avec l'espoir de s'emparer de l'objet de leur haine, fouillèrent mais en vain le pauvre petit couvent des Sœurs Grises de Saint-Norbert ${ }^{39}$, 》

Et Sara, la petite Sœur Grise missionnaire en la lointaine Île-à-laCrosse depuis mai 1871, par quelles angoisses passa-t-elle, loin des siens ?

37 Sœur Curran à la Maison Mère, 26 juillet 1870.

38 Archives, Maison P. S.B.

39 Le Métis, $3^{\circ}$ année, $n^{\circ} 21$. 
Elle disparut heureusement avant les sombres jours de 1885. Elle n'avait que 35 ans.

Elles vinrent, ces heures tragiques où Louis fut victime de la haine tenace de ceux qu'il avait tenus en échec.

Monseigneur Taché apprenant la triste nouvelle de l'exécution, rapportent nos chroniques, pria de suite $M$. Georges Dugas d'aller l'annoncer à la famille Riel, et de leur donner toutes les consolations possibles dans cette pénible circonstance.

Ce même jour, lundi 16 novembre, notre bonne Mère Vicaire Lamy, se fit elle aussi un devoir de porter à la mère et la veuve de la victime, les sincères sympathies de notre communauté et les assurer de nos plus ferventes prières.

Le lendemain, mardi, M. Georges Dugas, est allé dire la messe dans la chapelle de l'école de Saint-Vital. Les dames Riel assistèrent à cette messe et reçurent la sainte communion, pour se fortifier dans leur grande douleur $\mathbf{4 0}$.

Au cimetière de la cathédrale de Saint-Boniface, une simple colonne, entourée de verdure, marque la tombe de Riel dont la tâche est terminée.

\section{Conclusion}

Et voilà ce qu'ont relaté nos vieilles chroniques sur les événements que nous commémorons cette année. Nous avons décrit la vie de nos sœurs dans ce pays neuf de la Rivière Rouge et montré comment elles restèrent fidèles à ce petit peuple que monseigneur Provencher leur avait confié en 1844; nous avons même fortement appuyé sur leurs rapports avec les Riel.

Avec l'année 1870 , se termine l'âge héroïque, tout va maintenant se transformer autour d'elles. Elles continueront à enseigner dans les écoles de plus en plus nombreuses, à recueillir vieillards et orphelines, mais les visites à domicile en charrette cahotante s'espaceront de plus en plus. Désormais, elles recevront leurs malades dans leur hôpital. bien modeste l'hôpital de quatre lits de 1871, mais sa fondation marque tout de même le début d'une ère nouvelle.

Leur cher pays d'adoption est envahi par des gens de toute race et de toute langue et leurs chers Métis semblent se retirer dans l'ombre devant ce flot sans cesse grandissant.

Terminons par le mot de la sœur Curran: "N'est-il pas visible que Louis Riel fut l'élu de Dieu pour sauver son pays? »

Sœur Élisabeth DE MoIssaC. B.A.. M.A.. archiviste et bibliothécaire,

Maison Provinciale des Saurs Grises, Saint-Boniface, Manitoba.

40 Chroniques, Maison P. S.B., novembre 1885. 